\title{
DFT Study of Octanuclear Molecular Chromium-Based Ring Using New Pseudopotential Parameters
}

\author{
B. Brzostowski ${ }^{a}$, T. Ślusarski ${ }^{b}$ And G. Kamieniarz ${ }^{b}$ \\ ${ }^{a}$ Institute of Physics, University of Zielona Góra, Zielona Góra, Poland \\ ${ }^{b}$ Faculty of Physics, A. Mickiewicz University, Poznań, Poland
}

\begin{abstract}
We present a comprehensive study of magnetic properties of $\mathrm{Cr}_{8} \mathrm{~F}_{8}(\mathrm{Piv})_{16}$ molecular ring (in short $\mathrm{Cr} 8$ ) using the package SIESTA with several choices of chromium pseudopotential parameters. We use generalized gradient approximation to investigate properties of $\mathrm{Cr} 8$ ring approximated by replacing the pivallic group by $\mathrm{H}$ atoms (hydrogen saturation). For different choice of chromium pseudopotential we examine the electronic and magnetic properties of $\mathrm{Cr} 8$ molecule. We compare highest occupied molecular orbital and the lowest unoccupied molecular orbital gaps for both spin channels, magnetic moments, and exchange interaction parameter $J$. The influence of pseudopotential parameters on obtained results is discussed. Finally our results are compared with other theoretical approaches and experimental data.
\end{abstract}

PACS: 75.50.Xx, 71.15.Mb, 73.22.-f, 75.30.Et

\section{Introduction}

Computational methods in fields of quantum chemistry, biophysics or nanosized systems become one of the main approaches in understanding and predicting of properties of such systems. Due to rapid growth of computer technology and therefore accessing to treat more complex systems with more sophisticated methods, those simulations established themselves in strong position. When one thinks about understanding physical system behavior from principal quantum concepts, fundamental limitations automatically occur. Since we can analytically treat many-body problem only with strong approximations and in specific cases, the numerical solutions has clearly became more attracted but with risk of possibly leakage of physical intuition. Among many approaches, density functional theory (DFT) has become the method of choice for many large atomic systems. Magnetic molecules with coupled transition metal ions are interesting atomic systems that could be investigated using standard DFT approach. Family of magnetic wheels containing chromium paramagnetic centers is fairly promising in researching areas such as magnetic memories or magneto-electronics. From antiferromagnetic $\mathrm{Cr} 8$ molecule one can obtain a doped one, with nonzero magnetic moment, that could be attached to surfaces or linked into molecular dimer as a qubit candidate [1]. Such systems could be efficiently treated with DFT by such codes as SIESTA [2,3]. Despite they are relatively small systems when we think about calculations in real physical environment it is clear that we must pursue to maximal simplification in most basic case of isolated molecule without losing its physical properties. The major approach in this kind of problems is pseudopotential method. It allows us to get rid of many degrees of freedom of wave function in the core region. Since to get proper electronic structure we need to know only behavior of valence states in middle-atomic areas, we must also notice the necessity of inclusion semicore states in case of transition metals.
Despite there is no well known criteria of validity of chosen pseudopotentials they are much common for quality and time of calculations. Results obtained within them should be carefully examined with respect to similar problems treated with all-electron methods.

In this paper we study the problem of choice of pseudopotential generated within improved Troullier-Martins $[4,5]$ scheme in the case of chromium exchange-coupled systems. The testing procedure has two steps:

- testing trial excited states with fractional charge redistributions in free chromium atom to mimic the behavior of valence states in solid-like phase environment. Two main criteria are taken into account - the difference of energies of real and pseudo-valence state and the smoothness of pseudo-function (however the second is less important for SIESTA approach).

- putting chosen pseudopotentials fulfilling tests mentioned above into the bulk crystal and a molecule and how it will affect such a basic properties of this real systems like cohesion energy or magnetic moment and magnetic coupling.

Since in the ATOM [6] program for generating and testing pseudopotentials there is no possibility of including spin-orbit coupling the only relativistic contribution is the scalar correction of energy but only in the case of core electrons. But since chromium is light mass atom and in molecule compound there is almost frozen orbital momentum we have not found it as awkwardness. More important problem, when one thinks about getting reliable magnetic properties, is the amount of overlapping between core and semicore electrons. Due to nonlinearity of exchange-correlation potential we cannot separate them from each other. This effect is compensated through some excessive additional charge in core area technically followed from the core correction radius parameter. 


\section{Results and discussion}

As a first step we generated and tested thousands of pseudopotentials for $\mathrm{Cr}$ atom. For this purpose we have used the ATOM program changing the core matching radii for $4 s, 4 p, 3 d$, and $4 f$ orbital pseudowave functions in wide range up to 2.8 Bohr. For $d$ and $f$ channels smallest considered radius value was 0.7 Bohr, while for $s$ and $p$ channels smallest radius was just after last node of all electron wave function i.e. 1.7 Bohr and 1.8 Bohr, respectively. In our study we also change core corrections radius in the range of 0.5 to 1.5 and we found that the value 0.7 gives the best results. We also noticed that changing the core corrections radius in tested range slightly affects the results for the $\mathrm{Cr} 8$ molecule, but it is very important to take into account these corrections.

We generated all pseudopotentials for $\mathrm{Cr}$ with $4 s^{1} 3 d^{5}$ atomic configuration. On the other hand, pseudopotential is supposed to be transferable and provide an adequate description of an atom if the valence electronic configuration is varying in reasonable limits. Thus for all generated pseudopotential we compared results for atom with pseudopotential to the all-electron ones for 8 different trial configuration shuffling electrons between different $l$-channels, including fractional flow of electrons. Taking an all-electron calculation as a reference, we check for pseudopotentials if energies of valence states, and relations between total energies of different trial configurations are close to that all-electron one.

We obtain the best results of our tests for pseudopotential denoted as hard with matching core radii and core correction radius given in Table I. On the contrary, pseudopotential denoted as soft with rather large matching radii (cf. Table I) is less accurate and of course less computationally demanding. It still can give good results, but they should be treated with caution, rather as qualitative results. Another choices of pseudopotentials to compare are from our previous study of $\mathrm{Cr} 8$ properties $[7,8]$ — denoted as jnn, from paper by Santos et al. [9] denoted as njp. The last one, only for educational purpose, from SIESTA database (ABINIT $\rightarrow$ SIESTA conversion database) without core corrections - denoted as default. Parameters of these pseudopotentials are also given in Table I.

\section{TABLE I}

Matching core radii for chosen pseudopotentials. All in units of Bohr.

\begin{tabular}{c|c|c|c|c|c}
\hline \hline & Hard & Soft & jnn & njp & Default \\
\hline$r_{s}$ & 1.70 & 2.60 & 2.50 & 2.51 & 2.57 \\
$r_{p}$ & 2.00 & 2.80 & 2.50 & 2.80 & 2.57 \\
$r_{d}$ & 1.80 & 2.60 & 1.00 & 1.46 & 2.39 \\
$r_{f}$ & 1.00 & 2.60 & 1.00 & 1.00 & 2.39 \\
$r_{\text {core }}$ & 0.70 & 0.70 & 0.70 & 0.65 & -
\end{tabular}

Next we study properties of bulk chromium bcc crystal with selected pseudopotentials. We calculate cohesion energy taking into account basis set superposition error (BSSE) [10] correction (difference between the total energy of the atom in the bulk and single atom with the ghost atoms around it in the same geometry as in bulk). Including of such unoccupied states increases the variational freedom and the lowered energy is more accurate. For soft, jnn, and njp pseudopotentials we obtain $3.116,3.527$, and $3.461 \mathrm{eV}$ which is close to other theoretical results i.e. $3.58 \mathrm{eV}$ [11] and experimental data $4.1 \mathrm{eV}$ [11]. For hard pseudopotential we obtain value around $1 \mathrm{eV}$, while for default pseudopotential close to $0.3 \mathrm{eV}$. The deviation from the experimental value can be expected for the default pseudopotential, but for the hard pseudopotential is puzzling. We have checked that it persists even for extended (triple- $\zeta$ polarized, triple- $\zeta$ double polarized) bases and cutoffs.

Finally second test of pseudopotential in real systems is chromium wheel molecule. We carry out the SIESTA calculations using exchange-correlation potential proposed by Perdew, Burke, and Ernzerhof (PBE) [12] and exploit the same geometrical structures as those in [7, 13]. For all Siesta calculations the double- $\zeta$ polarized (DZP) basis set has been used as a good compromise of high accuracy and reasonable computational time. The resulting $k$-grid has been chosen according to the method of Monkhorst and Pack [14] using a $5 \times 5 \times 5 k$-grid. For all choices of pseudopotential we consider only ferromagnetic (FM) and antiferromagnetic (AFM) spin configurations of the $\mathrm{Cr}(\mathrm{III})$ ions in $\mathrm{Cr} 8$ molecule.

By comparing the total energies, we obtain in agreement with experiment that the AFM configuration corresponds to the ground state for four pseudopotentials. On the contrary, for default pseudopotential the FM state is ground state. It is clearly seen that the proper magnetic type of the ground state (AFM) is determined by taking into account the core corrections (cf. matching radii for default and soft pseudopotentials in Table I).

We estimate the magnetic coupling $J$ from the difference between the energies of the ground state and the excited configuration [13]. In Table II the total energy difference $\Delta E=E_{\mathrm{FM}}-E_{\mathrm{AFM}}$ and the corresponding values of exchange interaction parameter $J$ for different pseudopotentials are presented.

TABLE II

Exchange interaction parameter $J$ and total energy difference between the FM and AFM configurations for different pseudopotentials. All in units of meV.

\begin{tabular}{c|c|c|c|c|c}
\hline \hline & Hard & Soft & jnn & njp & Default \\
\hline$J$ & 6.441 & 6.287 & 6.598 & 6.594 & -0.683 \\
$E_{\mathrm{FM}}-E_{\mathrm{AFM}}$ & 231.881 & 226.320 & 237.532 & 237.385 & -24.599
\end{tabular}

We also calculate the local magnetic moments localized on the $\mathrm{Cr}$ (III) ions. DFT calculations based on the integration inside the muffin-tin sphere $[13,15]$ lead to the quantitative estimate $2.7 \mu_{\mathrm{B}}$, whereas the experiments and model calculations $[13,16-19]$ give the value $3 \mu_{\mathrm{B}}$. Our results are calculated by the Mulliken analysis, and are presented in Table III. These values agree with those found in $[13,15]$ for all pseudopotential choice but not for default pseudopotential. We should note that for hard, jnn, and njp pseudopotentials results are very close to the results from all electron study [15] and results for less accurate soft pseudopotential are surprisingly good. 
TABLE III

HOMO-LUMO majority and minority gaps and $\mathrm{Cr}$ magnetic moments for the $\mathrm{Cr} 8$ molecule in the AFM configuration.

\begin{tabular}{c|c|c|c|c|c}
\hline \hline \multicolumn{5}{c}{ AFM } \\
\hline & Hard & Soft & jnn & njp & Default \\
\hline$E_{\mathrm{H}-\mathrm{L}}^{\mathrm{maj}}[\mathrm{eV}]$ & 2.43 & 2.57 & 2.47 & 2.47 & 3.56 \\
$E_{\mathrm{H}-\mathrm{L}}^{\mathrm{min}}[\mathrm{eV}]$ & 2.37 & 2.50 & 2.41 & 2.40 & 3.47 \\
$\mathrm{~m}_{\mathrm{Cr}}^{\mathrm{Mul}}\left[\mu_{\mathrm{B}}\right]$ & -2.887 & -2.938 & -2.887 & -2.896 & +3.222 \\
& +2.877 & +2.926 & +2.876 & +2.884 & -3.207
\end{tabular}

Also highest occupied molecular orbital-lowest unoccupied molecular orbital (HOMO-LUMO) gaps for both spin channels are given in Table III. In AFM state HOMO-LUMO gaps $E_{\mathrm{H}-\mathrm{L}}^{\mathrm{maj}}$ and $E_{\mathrm{H}-\mathrm{L}}^{\min }$ for majority and minority spin channel are almost the same and all results are in good agreement with other theoretical investigations $[13,15]$, but again not for default pseudopotential.
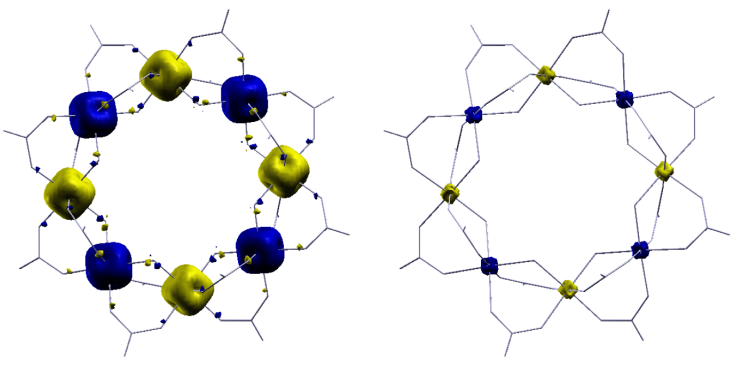

Fig. 1. Plot of the spin polarization of the charge density. Yellow (blue) shades are positive (negative) isosurfaces for the value 0.015 (left) and 0.4 (right) electrons / Bohr $^{3}$.

In Fig. 1 the three-dimensional spin polarization isosurfaces are shown. A significant spin polarization of the charge density is found only close to the $\mathrm{Cr}$ atoms. Both pictures in Fig. 1 are obtained for jnn pseudopotential. For a wide range of charge density values the hard and njp pseudopotential spin density plots are very similar to jnn one, while for default and fast pseudopotential these plots are slightly different but still similar to other one. In particular, the biggest differences in spin density plots for the different pseudopotentials are clearly seen for larger values of charge density (greater than 0.1 electrons $/$ Bohr $^{3}$ ), where soft and default pseudopotentials fail.

\section{Conclusions}

We have carried out the systematic study of influence of pseudopotential choice on $\mathrm{Cr} 8$ magnetic molecule properties. We have shown that taking into account core corrections is very important for magnetic structures. There is clearly shown that appropriate choice leads to very good results. Hard pseudopotential does not improve results for the molecule in a significant way and fails in the estimate of cohesion energy. Soft pseudopotential can give satisfactory results for the molecule, but should be treated carefully.

\section{Acknowledgments}

The numerical computations were performed in the PSNC Supercomputing Center in Poznań. This work has been supported by the grant No. N N202 230137 from the Ministry of Science and Higher Education (Poland).

\section{References}

[1] M. Affronte, F. Troiani, A. Ghirri, S. Carretta, P. Santini, V. Corradini, R. Schuecker, C. Muryn, G. Timco, R.E. Winpenny, Dalton Trans., 2810 (2006).

[2] P. Ordejón, E. Artacho, J.M. Soler, Phys. Rev. B (Rapid Commun.) 53, R10441 (1996).

[3] J.M. Soler, E. Artacho, J.D. Gale, A. García, J. Junquera, P. Ordejón, D. Sánchez-Portal, J. Phys., Condens. Matter 14, 2745 (2002).

[4] N. Troullier, J.L. Martins, Phys. Rev. B 43, 1993 (1991).

[5] N. Troullier, J.L. Martins, Phys. Rev. B 43, 8861 (1991).

[6] ATOM, a program for DFT calculations in atoms and pseudopotential generation, distributed as part of the SIESTA software package. See http:// www.icmab.es/siesta/atom .

[7] T. Ślusarski, B. Brzostowski, D. Tomecka, G. Kamieniarz, J. Nanosci. Nanotechnol. 11, 9080 (2011).

[8] T. Ślusarski, B. Brzostowski, D. Tomecka, G. Kamieniarz, Acta Phys. Pol. A 118, 967 (2010).

[9] E.J.G. Santos, A. Ayuela, D. Sánchez-Portal, New J. Phys. 12, 053012 (2010).

[10] It was firstly pointed out in H.B. Jansen, P. Ross, Chem. Phys. Lett. 3, 140 (1969), although the terminology BSSE was first introduced in B. Liu, A.D. McLean, J. Chem. Phys. 59, 4557 (1973).

[11] P.H.T. Philipsen, E.J. Baerends, Phys. Rev. B 54, 5326 (1996).

[12] J.P. Perdew, K. Burke, M. Ernzerhof, Phys. Rev. Lett. 77, 3865 (1996).

[13] D.M. Tomecka, V. Bellini, F. Troiani, F. Manghi, G. Kamieniarz, M. Affronte, Phys. Rev. B 77, 224401 (2008).

[14] H.J. Monkhorst, J.D. Pack, Phys. Rev. B 13, 5188 (1976).

[15] V. Bellini, A. Olivieri, F. Manghi, Phys. Rev. B $\mathbf{7 3}$, 184431 (2006).

[16] S. Carretta, J. van Slageren, T. Guidi, E. Liviotti, C. Mondelli, D. Rovai, A. Cornia, A.L. Dearden, F. Carsughi, M. Affronte, C.D. Frost, R.E.P. Winpenny, D. Gatteschi, G. Amoretti, R. Caciuffo, Phys. Rev. B 67, 094405 (2003).

[17] G. Kamieniarz, P. Kozłowski, G. Musiał, W. Florek, M. Antkowiak, M. Haglauer, A. Caramico d'Auria, F. Esposito, Inorg. Chim. Acta 361, 3690 (2008).

[18] P. Kozłowski, G. Kamieniarz, M. Antkowiak, F. Tuna, G.A. Timco, R.E.P. Winpenny, Polyhedron 28, 1852 (2009).

[19] S. Piligkos, H. Weihe, E. Bill, F. Neese, H. El Mkami, G.M. Smith, D. Collison, G. Rajaraman, G.A. Timco, R.E.P. Winpenny, E.J.L. McInnes, Chem. Eur. J. 15, 3152 (2009) 